Ich füge nur noch hinzu, daß die diphthongischen Formen von $i \bar{u} s$ und Ableitungen wie ious, ex ioure, ioudex, ioudicent $u$. a. in den Fragmenta legis Serviliae repetundarum, restituit Klenze Berolini 1825 sehr häufig sind.

Wien.

v. Grienberger.

\title{
Zum Wandel des idg. $o$ im Germ.
}

Bereits IF. 15, 129 Fußn. habe ich zu v. Wijks Aufsatz 'Zur relativen Chronologie urgermanischer Lautgesetze', PBrB. 28, $243 \mathrm{ff}$., Stellung genommen und mich seinen Ausführungen im Wesentlichen angeschlossen. Doch galten meine damaligen Bemerkungen nur der Methode v. Wijks; was dagegen seine Schlußfolgerungen für die Chronologie der germ. Vokalentwickelung betrifft, so hat er hier noch nicht das letzte Wort gesprochen, um so weniger, als etwa gleichzeitig mit dem genannten Aufsatz ein solcher von Bremer erschien, IF. 14, 363 ff., der mit v. Wijk keineswegs übereinstimmt. Während dieser einen Wandel des idg. $o$ im Germ. in 3 verschiedenen Perioden annimmt: 1. haupttonig zu $a$, 2. nichthaupttonig zu $a$ 'außer in einigen wenigen Fällen', 3 . in diesen wenigen Fällen zu $u$ (s. S. 252 f.), kommt Bremer zu dem Schluß, daß im Germ. in vorchristl. Zeit jedes idg. o zu $a$ geworden ist, wohl in sämtlichen Stellungen zu gleicher Zeit. Man kommt bei einem Vergleich beider unwillkürlich auf den Gedanken, daß vielleicht, wie meist bei 2 schroff gegenüberstehenden Meinungen das Richtige in der Mitte liegt, so auch der fragliche Wandel von $o$ weder dreimal, noch einmal, sondern eben zweimal stattgefunden hat.

Zunächst das zeitliche Verhältnis des haupttonigen und nichthaupttonigen Überganges von $o$ in $a$. Wie Bremers Zusammenstellung zeigt, hat vor ihm überhaupt niemand daran Anstoß genommen, daß der erstere älter ist; und zwar ist der Anlaß zu dieser herrschenden Ansicht die Tatsache, daß in den uns überlieferten Brocken aus urgerm. und ältester dialektischer Zeit sich zwar zahlreiche Fälle von nichthaupttonigem $o$ finden, wie Ariovistus, Inguiomerus, Teutoburgiensis (s. die Beispiele bei Bremer), aber kein einziger Beleg von haupttonigem o. Dazu kommt die im Prinzip zweifellos richtige Beobachtung von Hirt, 
PBrB. 23, 317 : die kelt. Lehnwörter wie Maginza, Wascono usw., die $a$ statt des haupttonigen $o$ haben, beweisen nicht, daß diese Entlehnungen älter als der Wandel des haupttonigen $o$ sind, denn a kann hier durch Lautsubstitution für einen im Germ. nicht vorhandenen Laut eingetreten sein.

Nun muß aber berücksichtigt werden, daß Hirts Substitutionsprinzip die vorliegende Frage garnicht fördert; denn es ist ja damit nicht gesagt, daß der Wandel älter sein muß als die Entlehnungen, sondern nur, daß er es kann. Außerdem aber scheint es mir überhaupt gewagt, die Substitutionstheorie bei den genannten Entlehnungen anzuwenden. Denn angenommen, daß der haupt- und nichthaupttonige Wandel des $o$ zeitlich auseinanderliegen, so wäre ja erforderlich, daß auch der letztere bereits stattgefunden hätte zur Zeit der Entlehnung, sowohl im allgemeinen als auch in den "wenigen, besonderen Fällen", während andererseits der $a$-Umlaut des $u$, der ja wieder ein neues $o$ schuf, noch nicht eingetreten sein durfte; denn nur in dieser vermutlich sehr kurzen Spanne Zeit zwischen der von v. Wijk mit III bezeichneten Periode und dem $a$-Umlaut, die übrigens beide als nicht urgerm. feststehen, gab es in den germ. Dialekten kein $o$, während sonst wenigstens in nichthaupttonigen Silben sicher dieser Vokal vorkam. Ich glaube daher, daß Hirts Ausführungen nicht ohne Grund von Kluge, Pauls Gr. $1^{2}, 414$ und von Bremer mit Stillschweigen übergangen werden, und daß wir mit Streitberg und anderen den freilich nicht absolut bestimmbaren Zeitpunkt der Entlehnungen als terminus, post quem für den Lautwandel anzusehen haben.

Folgende Momente nun sprechen stark dagegen, daß der Wandel von $o$ in $a$ haupttonig früher stattfand als nichthaupttonig:

1. In den finnischen Lehnwörtern zeigt sich nicht nur vom haupt-, sondern auch vom nichthaupttonigen $o$ keine Spur mehr.

2. Wie finn. rengas zeigt, ist der Wandel des nichthaupttonigen $o$ älter als der von $e$ zu $i$ vor $r+$ Kons., letzterer ist aber nach Bremer IF. 4, 18, 30 bereits ins 1. Jahrh. v. Chr. zu setzen ${ }^{1}$ ), da er älter ist als vor $n, m$

1) Helm, PBrB. 23, 555 ff. erblickt in dem durch Cäsar überlieferten Tencteri den älteren Zustand, wohl mit Recht; aber das steht mit Bremer nicht in Widerspruch, da dann nur das Jahr $55 \mathrm{v}$. Chr. als terminus, post 
+ Kons. Der nichthaupttonige Wandel von o zu $a$ darf also nicht zu spät angesetzt werden.

3. Andererseits darf der Wandel des haupttonigen $o$ keiner zu frühen Periode des Urgerm. zugeschrieben werden, falls Möller, PBrB. 7, 483 mit der Ansicht recht hat, daß in der verschiedenen Behandlung der Labiovelare noch eine Spur der Verschiedenheit von idg. $o$ und $a$ zu erblicken ist ${ }^{1}$ ).

Nun bleiben aber die zahlreichen, aus urgerm. Zeit überlieferten Wörter, meist Namen, übrig, die tatsächlich nur nichthaupttoniges $o$ zeigen, und denen lediglich die Ansicht zu verdanken ist, daß sich dieses länger erhielt als das haupttonige. Von diesen Belegen scheiden aber die aus, die nach der von v. Wijk unter III angegebenen Weise behandelt wurden, wo also der Wandel zu $a$ lautgesetzlich unterblieb. Welches sind nun die Bedingungen, unter denen dies geschah? Brugmann nennt 1. folgendes, nicht ursprünglich auslautendes $m, 2 . u$ der Folgesilbe. Wie wäre es denn, wenn man bei dem ersten Punkt statt $m$ jeden Labial setzt? v. Wijk deutet dies an, ohne näher darauf einzugehen. Wie ich sehe, sind dann fast alle Beispiele für erhaltenes nichthaupttoniges 0 als lautgesetzlich anzusehen. Ein Blick auf das von Bremer IF. 14, 363 ff. gebrachte Material lehrt das; ich erinnere nur an Langobardi, Hariobandus, Chariovalda, Maroboduus, Mallovendus usw. Die Wahrscheinlichkeit dieses Lautgesetzes wird dadurch gefördert, daß auch im Lat. und Osk. unbetontes $o$ in offener Silbe vor Labial anders als gewöhnlich behandelt wurde; siehe Sommer, L. u. Fl. 113, Brugmann, Grundr. $1^{2}, \S 145$.

Nun ist freilich nicht nur in historischer Zeit, sondern schon in dieser gem.-westgerm. Periode der ursprüngliche Zustand durch, übrigens naheliegende, Analogiebildungen stark verwischt worden; daran darf man sich nicht stoßen. Namensformen wie Alamanni können sehr wohl durch andere wie (got.) Alareiks beeinflußt sein, umgekehrt kann as. alohel auf Anlehnung an alomahtig beruhen (s. u.); auch können wir vielfach trotz Streitberg, Gr. $46 \mathrm{f}$. Einfluß von kelt. Namensformen annehmen, wie es

quem gilt. Nur darf man nicht, wie Bremer S. 18 will, bis ins 2. Jahrh. v. Chr. hinaufgehen.

1) Möllers Ansicht wird von den meisten Gelehrten geteilt: Streitberg, Urg. Gr. 46 f., Brugmann, Gr. $1^{2}, \S 147,1 ; 678$, Kluge, Pauls Gr. $1^{2}, 374$. Neuerdings aber ist Streitberg, wie ich brieflich von ihm erfahren, zweifelhaft geworden. 
Bremer tut; nur möchte ich das auf solche Formen beschränken, bei denen das zweite Glied auch in kelt. Wörtern begegnet, z. B. Cruptorix, Malorix, $\Delta \epsilon \cup \delta o ́ p ı$ nach Dumnorix, Orgetorix, denn im Lat. war 0 als Zwischenvokal nicht gebräuchlich ${ }^{1}$ ).

Ich will hier nicht das ganze Material des West- und Nordgerm. besprechen, das würde eine viel zu umfangreiche und dabei fruchtlose Untersuchung sein, fruchtlos deshalb, weil in den meisten Dialekten schon frühzeitig die qualitative und quantitative Schwächung der unbetonten Vokale beginnt; und einem mittleren $e$ sieht man nicht an, ob es aus $a$ oder aus $o$ resp. $u$ entstanden ist, ebensowenig kann man eine Entscheidung treffen, wenn der unbetonte Vokal zu Null geschwächt ist. Aber einiges möchte ich hervorheben.

Ich greife die uns sonst nur ganz trümmerhaft überlieferte Sprache der Langobarden heraus, da das reiche Material an Eigennamen, das sie bietet, von Bruckner, Quell. u. Forsch. 75, 215-326 ausgezeichnet geordnet und erklärt ist. Über die Schicksale der unbetonten Vokale handelt Bruckner a. a. 0.113 ff. Er stellt dabei fest, daß das $a$ der Mittelsilben in der Komposition bereits früh zu $e$ und sogar zu Null geschwächt wurde. Am lehrreichsten sind dabei immer solche Formen, denen ein ursprünglicher Typus eines anderen Dialektes gegenübersteht, z. B. Sinderadus - ahd. Sindarat. Andererseits ist in zahlreichen Fällen mittleres $o$ bezw. $a$ erhalten, und hier sagt Bruckner wohl richtig, daß wir es bei der Mehrzahl dieser Namen, wie Alaricus, Ródoricus, deutlich mit einer Assimilation des unbetonten und unbestimmten Themarokals an den hochbetonten Stammrokal, also mit etwas Unursprünglichem, zu tun haben. Bei einigen Fällen aber versagt diese Erklärung: Langobardi, Tancomarus, Dacopertus (belegt mit $u$, s. S. 115), von denen besonders letzteres wegen ahd. Dagobert alt sein dürfte.

In einem Punkt aber scheint mir Bruckners Auffassung nicht das Richtige zu treffen, nämlich bez. der Kompp., deren zweites Glied mit $w$ anlautet: -wald (S. 317 f.), -ward (319), -war (319),

1) Prinzipiell ist die Möglichkeit einer solchen Angleichung durchaus anzuerkennen. Im Griech. z. B. sind Kompp. mit dem Bindevokal o

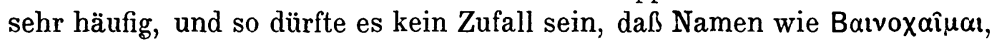
Baıтó punkt unverständlich ist, gerade durch griech. Überlieferung erhalten sind. Was Theodoricus betrifft, so bezweifelt wohl niemand die volksetymologische

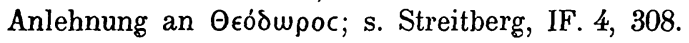


-win (323), z. B. Dacoald, Aloardus, Aloara, Albuin. Bruckner nimmt $129 \mathrm{f}$. an, daß bei allen solchen Zusammensetzungen der stammbildende Vokal des ersten Gliedes geschwunden und das anlautende $w$ des zweiten Gliedes zu $o, u$ (diese beiden sind ziemlich gleichwertig) vokalisiert ist, wie wir das ja auch an anderen Stellen des germ. Sprachgebietes kennen. Nun mußte Bruckner aber annehmen, daß diese Vokalisierung erst vollzogen wurde, nachdem in manchen Fällen der Stammbildungsvokal synkopiert war, und daß dann nachträglich, wiederum nur in manchen Fällen, das aus $w$ neu entstandene $o$ schwand; denn wir haben durchaus nicht selten Formen wie Grîsaldus, Taraldus, Sudbînus, besonders seit dem Ende des 8. Jahrhs. Da scheint mir doch die Entwicklung einfacher, daß erst das zwischenvokalische $w$ lautgesetzlich überall schwand, daß also ${ }^{*}$ Dacowald zu Dacoald wurde, und daß Grîsaldus usw. ebenso wie z.B. Teutprandus oder ahd. Diotmar zu beurteilen sind. Die vorkommenden Schreibungen Albwini, Odwini, die etwa gegen meine Ansicht sprechen könnten, werden von Bruckner selbst als nicht beweiskräftig angesehen. Dagegen ist der älteste Typus z. B. in Adlowaldus erhalten. Der erste Schritt bestand wohl darin, daß $w$ zu $h$ geschwächt wurde, eine Stufe, die man vielleicht in den Formen Albuhin, Audohin erblicken kann. Erst allmählich ging dann die Artikulation dieses Hauchlautes ganz verloren. Denn auch das aus urgerm. $\chi$ entstandene $h$ schwand zwischen Vokalen, und es ist lehrreich, daß in den Zusammensetzungen mit-hard, -hari im zweiten Glied (s. S. 265 f.) nicht ein einziger Fall von -oardus, -oari vorliegt, sondern daß hier der Mittelvokal, der nur $a$ sein konnte, vermutlich durch Kontraktion mit dem folgenden $a$ verschmolzen wurde. Erhalten ist dies $h \mathrm{z}$. B. in Kebahartus.

Ein Blick in Förstemanns „Altdeutsches Namenbuch" lehrt, daß dieser Zustand des Lang. von den übrigen Dialekten geteilt wird, also durchaus etwas Altes ist. Man beachte Gundobalt, Gundobert, Gundofrid, Gundomero, Gundovald, Gundowin gegenüber Gundachar, Guntaswind usw., ähnlich Audofleda, Audomar, Audovald gegenüber Audagar. Allerdings sind auch hier die lautgesetzlichen Verhältnisse stark durchbrochen, aber die der Regel widersprechenden Formen sind fast immer die jüngeren und überdies meist durch Einfluß der umgebenden Vokale zu erklären, so bei Tagamar, das dem 9., gegenüber Dacomar, das dem 8. Jahrh. angehört; dasselbe zeitliche Verhältnis besteht bei Alabert-Alubert. 
Ganz deutlich zeigen diese Fernassimilation Namen wie Hadagast, Hadamar, deren erster Bestandteil ja ein $u$-Stamm war; beide sind jung, für letzteres ist das ältere Catumer (Tacitus) belegt.

Die besprochene Erklärung paßt, wie mir scheint, auch auf die as. Adjektiva alomahtig, alowaldand, alowaldo, denen sich dann alohel angeschlossen hat. Ich halte diese Auffassung für einfacher als die von Kluge, IF. 4, 311, der zu einem sonst nirgends belegten Stamm *alwa- seine Zuflucht nimmt.

Ich glaube also mit Bremer, daß nichts uns zwingt, in v. Wijks chronologischer Tabelle die Perioden I und II zu trennen, möchte aber mit $v$. Wijk als Bedingung für die Erhaltung des nichthaupttonigen $o$ die Stellung vor jedem Labial und vor $u$ der Folgesibe ansehen. Auch sonst bedarf die Tabelle v. Wijks der Berichtigung; z. B. liegt kein Grund vor, die Monophthongierung von ei so spät anzusetzen, da wir für den Diphthong. überhaupt keine Spuren mehr haben; s. Brugmann, Grundr. $1^{2}$, 189, Kluge, Grundr. $1^{2}, 415$. Auch der Wandel von $e$ zu $i$ vor Nas. + Kons. dürfte älter sein als der $i$-Umlaut des $e$. Vor gutturaler Gruppe ist er sogar sicher älter als Periode III, wie durch Inguiomerus bewiesen wird. v. Wijk übersah dabei, daß beide Erscheinungen, $e i: \bar{\imath}$ und $e r s: i r$ (s. o.) urgerm. sind, Periode III aber nicht, da das Ostgerm. nicht teilnimmt am Wandel von $o$ zu $u$. Von Periode IV ist also nur der $i$-Umlaut jünger als III, und dieser braucht ja auch nicht urgerm. zu sein, da gem.-ostgerm. jedes idg. $e$ zu $i$ wurde. Der $a$-Umlaut, der ja oben S. 36 auch in die Debatte gezogen werden mußte, ist jedenfalls jünger als PeriodeIII; sein Verhältnis zum $i$-Umlaut läßt sich nicht feststellen.

Leipzig.

Kurt Eulenburg.

\section{Múspell.}

König Gýlfi von Schweden wollte die Ásen kennen lernen; er wollte erfahren, wieso diese so mächtig wären, ob durch eigene Kraft, oder durch die Kraft der Götter, die sie verehrten - eine merkwürdige Perspektive, die den Göttern abermals Götter überordnet - doch wollte er nicht, daß seine Fahrt nach Ásgarđ offenkundig würde; deshalb nahm er, der zauberkundig war, die Gestalt eines alten Mannes an und machte sich auf den Weg. 